\title{
Sensitivity Analysis Based Optimization for Linear and Nonlinear Parameters in AVR to Improve Transient Stability in Power System
}

\author{
Seung-Mook Baek \\ Division of Electrical, Electronic and Control Engineering, \\ Kongju National University, Cheonan, Chungnam, South Korea \\ smbaek@kongju.ac.kr
}

\begin{abstract}
The paper describes an optimal parameter selection method of automatic voltage regulator (AVR) for a large power plant in order to improve power system stability. The $A V R$ is generally used to control the output voltage of the generator by regulating the excited voltage in a generator rotor. In order to improve transient stability in power system, the paper proposes the method to select optimal parameters of the AVR based on exact power system modeling. The performances of the proposed method are carried out by time-domain simulation by applying fault to a power system
\end{abstract}

Keywords: Automatic voltage regulator, optimization, parameter selection, sensitivity analysis, transient stability

\section{Introduction}

Recently, power systems have become large and complex and most controllers need faster and more accurate actions [1-3]. The automatic voltage regulator (AVR) is an important controller in power generator. The AVR is used for changing DC voltage in a rotor of a power generator. Therefore, it controls a terminal voltage of a generator in order to keep the system voltage constant and also generate reactive power to the system. It is a kind of high speed controller to achieve its purposes. Therefore, the appropriate selection of parameters of the AVR is very critical issue.

The dynamic behavior of the power system with the AVR is affected by the linear parameters (gain and time constants of phase compensator) and the constrained parameters (saturation output limits) resulting in nonlinear characteristic. The proper selection of linear parameters has been usually achieved based on linear approaches such as eigenvalue analysis with linearized model [4-5]. However, the output limit values cannot be tuned with such method. On the other hand, both of linear and nonlinear parameters of AVR have crucial effect on the operation and performance during transient situation. Therefore, optimal selection of both kinds of parameters is important to maintain power system stability. In order to optimize linear and nonlinear parameters of AVR, sensitivity analysis with respect to certain objective value is obtained with nonlinear modeling of power system. An exact power system modeling method is one of the best approaches in order to accomplish optimization technique with sensitivity analysis. In this paper, a method to optimize the linear and nonlinear parameters of the AVR with exact power system modeling is proposed and the performances are assessed by case study carried out on a single machine connected with infinite bus system.

The paper is organized as follows: Section 2 presents a summary what is AVR and its role in power system. Then, the exact modeling method for power system is described in Section 3 and hence the method to optimize the parameters of AVR is 
explained in Section 4. Section 5 shows the performances of the proposed method in the paper. Finally, the conclusions are given in Section 6.

\section{Automatic Voltage Regulator}

The AVR is designed to control the DC voltage in the generator rotor. By varying DC voltage of the rotor, the magnetic field in the generator is varied. This action brings the changes of real and reactive power output of the generator. Especially, the reactive power output is very closely related to the rotor DC voltage. Also, the change of reactive power output influences the terminal voltage of the generator and finally the AVR has an effect on the variation of the bus voltage in power system.

The AVR controller is given in Figure 1. It is accurately modeled and the power generator is equipped with the AVR. The AVR in Fig. 1 consists of four controllers, which are two low pass filters, phase-lead compensator, and output limits. Two filters are used to remove high frequency element of the flowing signal inside the AVR. The phase-lead compensators make the signal faster or slower. Finally, the output limits are used to prevent from flowing out very large signal from AVR.

Generally, for the AVR, the gain $\left(\mathrm{K}_{\mathrm{A}}\right)$ is set to 200. Also, it is typical values of $0.04,12,1,1.05,5$, and -5 for $\mathrm{T}_{\mathrm{R}}, \mathrm{T}_{\mathrm{B}}, \mathrm{T}_{\mathrm{C}}$, and $\mathrm{T}_{\mathrm{A}}$, respectively [6]-[7].

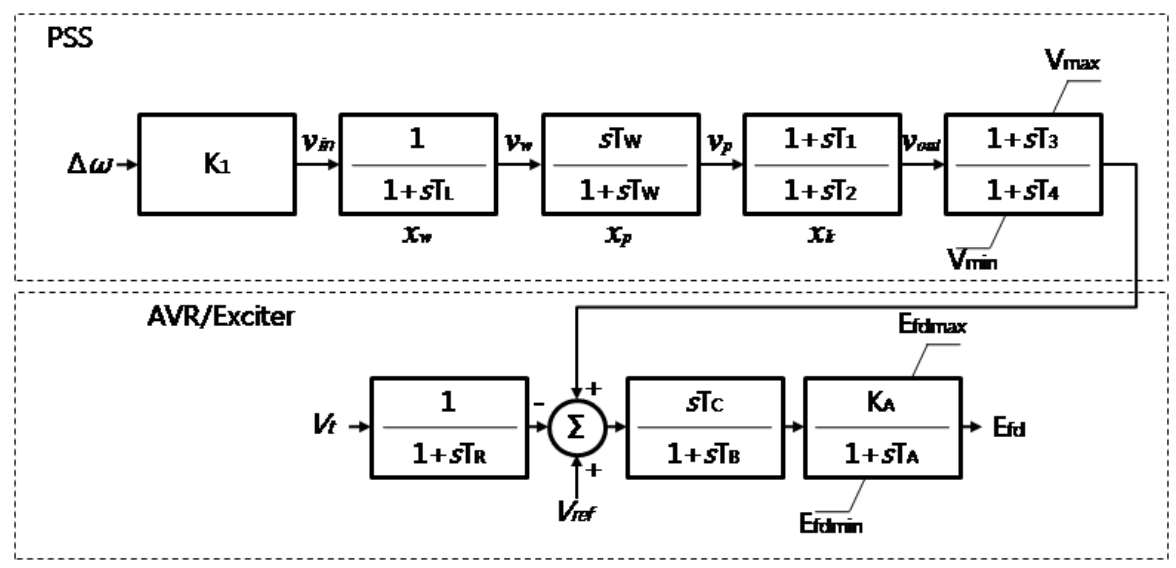

Figure 1. AVR Block Representation

The AVR in Figure 1 is connected to the generator and injects output signal to control the DC voltage of rotor in generator. In general, in order to evaluate the performance of the AVR, a simple power system in which the generator is connected to the infinite bus is considered as illustrated in Figure 2. The infinite bus means the connection point of the generator to very large power system. That means that even though the large changes occur in the generator, the effects to the large power system are very small. Therefore, this system is very useful in order to see the performances of various controllers for the power generator. The generator equipped with the AVR and a power system stabilizer (PSS) is connected to the infinite bus by transmission system and some disturbances are applied to bring oscillation to power system. 


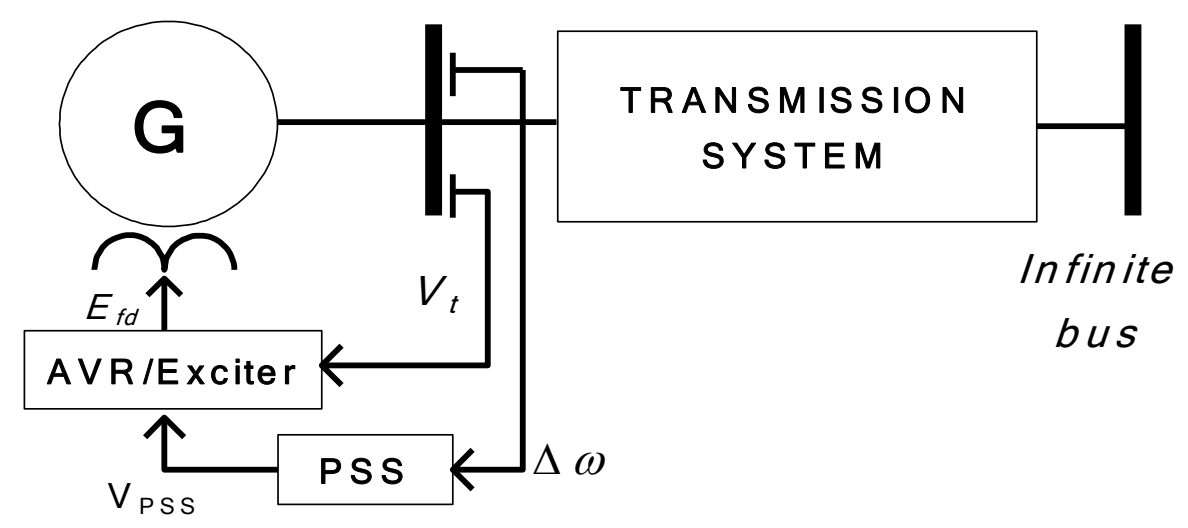

Figure 2. Single-Machine Connected to Infinite Bus (SMIB) System

\section{Power System Modeling}

As mentioned in the previous section, the behavior of the power system is characterized by the following:

- Continuous and discrete states.

- Continuous dynamics.

In other words, the power system needs to be modeled mathematically by consisting of the interactions between linear and nonlinear behaviors. Therefore, the power system modeling with the differential and algebraic equations can be presented without loss of generalities as follows [8].

$$
\begin{aligned}
& \dot{x}=\underline{f}(\underline{x}, y) \\
& 0=g(\underline{x}, y)
\end{aligned}
$$

where $x$ represents the continuous dynamic states, for example generator angles, speed, and fluxes; $y$ represents algebraic states, e.g., load bus voltage magnitudes and angles.

The flows $\phi$ in (3) (4) of a system will generally vary with changes in parameters and/or initial conditions. Trajectory sensitivity provides a way of quantifying the changes in the flow that result from (small) changes to parameters and/or initial conditions. The development of these sensitivity concepts will be based on the power system model in (1) (2). Trajectory sensitivities follow from the Taylor series expansion (neglecting higher order terms) of the flows $\phi_{\underline{x}}$ and $\phi_{y}$ in (3) (4), which can be expressed as

$$
\begin{aligned}
& \Delta \underline{x}^{(t)}=\Delta \phi_{\underline{x}}\left(\underline{x}_{0}, t\right) \approx \frac{\partial \phi_{\underline{x}}\left(\underline{x}_{0}, t\right)}{\partial \underline{x}_{0}} \Delta \underline{x}_{0} \equiv \boldsymbol{\Gamma}_{\underline{x}_{0}}(t) \Delta \underline{x}_{0} \\
& \Delta y(t)=\Delta \phi_{y}\left(\underline{x}_{0}, t\right) \approx \frac{\partial \phi_{y}\left(\underline{x}_{0}, t\right)}{\partial \underline{x}_{0}} \Delta \underline{x}_{0} \equiv \boldsymbol{\Gamma}{ }_{y}(t) \Delta \underline{x}_{0}
\end{aligned}
$$

where $\Gamma_{\underline{x}_{0}} \in \mathfrak{R}^{\underline{n} \times \underline{n}}$ and $\Gamma_{y} \in \mathfrak{R}^{m \times \underline{n}}$ are partial derivatives matrices of system flows and known as the trajectory sensitivities. Recall that $\underline{x}_{0}$ incorporates the parameters $\lambda$, therefore the sensitivities to initial conditions $\underline{x}_{0}$ include parameter sensitivities.

With Eqs. (1) (2), the power system can be modeled as maintaining its nonlinear properties. The generator $(\mathbf{G})$ in Figure 2 is accurately represented by a six-order machine model, viz., a two-axis $(d-q)$ model with two damper windings in each axis [9]. That means that six differential equations are needed to make mathematical model for the generator. More detailed explanations related to the SMIB system 
including the generator are given in [5]. Also, the differential and algebraic equations on the AVR and PSS in Figures 1, respectively, can be derived by Eqs. (5) (8). The parameters in these equations are indicated in each model in Figure 1. With this method, the whole system is analyzed and constructed by using Eqs. (1) (2), we can compute the trajectory sensitivities in order to solve optimization problems.

$$
\begin{aligned}
f_{p s s}=\left\{\begin{array}{l}
\dot{x}_{w}=v_{w} / T_{w} \\
\dot{x}_{p}=T_{2} v_{p}-T_{1} v_{w} \\
\dot{x}_{k}=T_{4} v_{p}-T_{3} v_{k}
\end{array}\right. \\
g_{p s s}=\left\{\begin{array}{l}
\Delta w K_{1}-v_{w}-x_{w} \\
T_{2} v_{p}-T_{1} v_{w}-x_{p} \\
T_{4} v_{\text {out }}-T_{3} v_{p}-x_{k}
\end{array}\right. \\
f_{A V R}=\left\{\begin{array}{l}
\dot{x}_{1}=1 / T_{R}\left(y_{3}-x_{1}\right) \\
\dot{x}_{2}=1 / T_{A}\left(K_{A} \cdot y_{6}-x_{2}\right) \cdot y_{10} \cdot y_{11} \\
\dot{x}_{3}=y_{5}-y_{6}
\end{array}\right. \\
g_{A V R}=\left\{\begin{array}{l}
g_{1}=y_{3}^{2}-\left(y_{1}^{2}+y_{2}^{2}\right) \\
g_{2}=T_{B} \cdot y_{6}-T_{C} \cdot y_{5}-x_{3} \\
g_{3}=y_{5}-\left(x_{3}+y_{4}-x_{1}\right) \\
g_{4}=y_{8}-x_{2}-E f d_{\text {max }} \\
g_{5}=y_{9}-x_{2}-E f d_{\text {min }}
\end{array}\right.
\end{aligned}
$$

\section{Optimization Technique}

In order to select linear parameters of common controllers, conventional linear methods such as eigenvalue analysis or bode plot can be simply applied. However, those methods need obligatorily linearized power system modeling. If the system is modeled linearly, linear parameter can be optimized. Otherwise, nonlinear parameter cannot be tunable. Besides, linearized model can sometimes degrade the performances of tuned parameters due to excessive simplification of the system [10].

In the paper, the power system with its controllers including the AVR is exactly modeled to represent nonlinear behavior. Therefore, the linear approaches cannot be easily applicable and nonlinear optimization technique is needed to find proper parameter set of linear and nonlinear parameter of the AVR.

Firstly, the given optimization problem is required to formulate a Bolza form of objective function, $\mathbf{J}$ in (1) to (3).

$$
\begin{aligned}
& \min _{\lambda, t_{f}} \mathbf{J}\left(\underline{x}, y, \lambda, t_{f}\right) \\
& \text { suject to } \left.\left[\begin{array}{l}
\underline{x}(t) \\
y(t)
\end{array}\right]=\phi\left(\underline{x}_{0}, t\right) \text {, where } \underline{x} \in S \text { (constraint } \quad \text { set }\right) \\
& \mathbf{J}=\varphi\left(\underline{x}^{(}\left(t_{f}\right), y\left(t_{f}\right), \lambda, t_{f}\right)+\int_{t_{0}}^{t_{f}} \psi(\underline{x}(t), y(t), \lambda, t) d t,
\end{aligned}
$$

where $\lambda$ are the optimized parameters (here, nonlinear parameter set which is optimally tuned), which are adjusted to minimize the value of $\mathbf{J}$ in (9), and $t_{f}$ is the final time during each optimization process. Also, $\varphi$ is the cost or penalty associated with the error in the terminal state at time $t_{f}$, and $\psi$ is the cost function associated with transient state errors. 
Then, the objective function, $\mathbf{J}$ is defined to mitigate system damping and force the system to recover to a post-disturbance stable operating point as quickly as possible. The speed deviation $(\Delta \omega)$ and terminal voltage deviation $\left(\Delta V_{t}\right)$ of the generator are considered as good assessments of the damping and recovery [11]. Therefore, the function $\mathbf{J}$ in (9) is re-formulated with specific time, $t_{f}$ as follows.

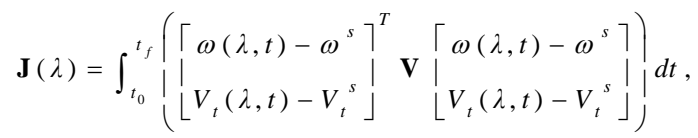

where $\mathbf{V}$ is the weighting matrix, and $\omega^{s}$ and $V_{t}^{s}$ are the post-fault steady state values of $\omega$ and $V_{t}$, respectively. Note that the diagonal terms in the matrix $\mathbf{V}$ are determined by considering the balance of conflicting requirements on the speed and voltage deviations [12].

Then, these parameters $\lambda$ are updated by using (13) with $\nabla \tilde{\mathbf{J}}(\lambda)$ during iteration. This is steepest descent method which is one of the common methods to optimize parameters in nonlinear systems.

$$
\lambda_{k+1}=\lambda_{k}+\alpha \cdot \nabla \tilde{\mathbf{J}}(\lambda)
$$

where $\alpha$ is the step-length.

At the end of each run, the convergence performance is evaluated by the userdefined criterion, which is the maximum relative changes in parameters $\left(S_{C}\right)$ as given in (14) as well as the value of $\mathbf{J}$. Note that the parameter optimization problem in the paper aims to minimize the value of objective function $\mathbf{J}(\lambda)$ with the small number of iterations.

$$
S_{C}=\left\|\frac{\lambda_{k+1}-\lambda_{k}}{\lambda_{k+1}}\right\|_{\infty} .
$$

Table I shows the initial and optimal parameters of AVR tuned in the proposed methods. The gain is much less than the initial value, this means that the large gain is not necessary to improve the transient performances. The time constants are not significantly changed but upper limit value is twice larger than initial value even though the lower value is not completely changed.

Table 1. Optimal Parameters of AVR

\begin{tabular}{|c|c|c|c|c|c|}
\hline Values & $\mathrm{K}_{\mathrm{A}}$ & $\mathrm{T}_{\mathrm{B}}$ & $\mathrm{T}_{\mathrm{C}}$ & $\operatorname{Efd}_{\max }$ & $\operatorname{Efd}_{\min }$ \\
\hline Initial & 200 & 12 & 1 & 5 & -5 \\
\hline Optimal & 97.95 & 11.30 & 2.68 & 11.97 & -5 \\
\hline
\end{tabular}

\section{Simulation Results}

In order to simulate the proposed control scheme, the paper uses Matlab software to build differential and algebraic equations for power system and its controllers including the AVR and PSS. Thereafter, a disturbance is applied to the terminal bus of the generator in Figure 2.

Power system in Figure 2 is disturbed by applying a $100 \mathrm{~ms}$ three-phase short circuit fault with the fault-impedance of 0.05 pu to the generator terminal bus at 0.1 s to see the transient stability. The proposed method can bring good performances on the transient stability as shown in Figures 3 to 5.

Figure 3 shows the rotor angle response of the generator during the disturbance. It is clearly shown in the figure that the oscillation is dramatically decreased after the second swing even though the variation with initial parameters lasts until the 
end of the simulation. This means that the system is stabilized within only 3 seconds after large disturbance if the optimal parameters in Table I are applied.

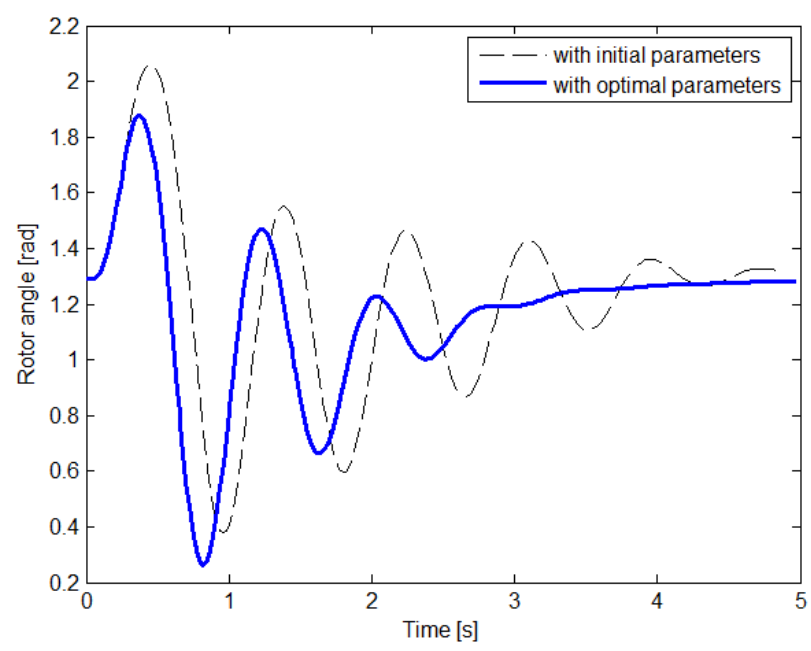

Figure 3. Rotor Angle Response [rad]

Figures 4 and 5 show the speed deviation and real power responses of the generator, respectively. The oscillations with optimal parameters are little bit larger than those with initial ones shortly after the disturbance. However, the swings are reduced right away within 3 second after the disturbance. Conclusively, the optimal parameters brings more stability in power system than initial ones.

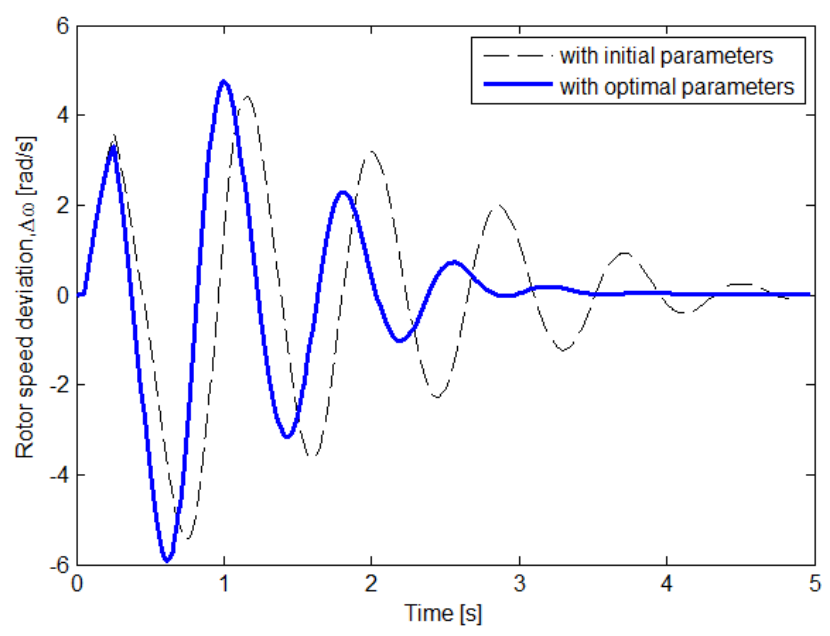

Figure 4. Generator Speed Deviation [rad/s] 


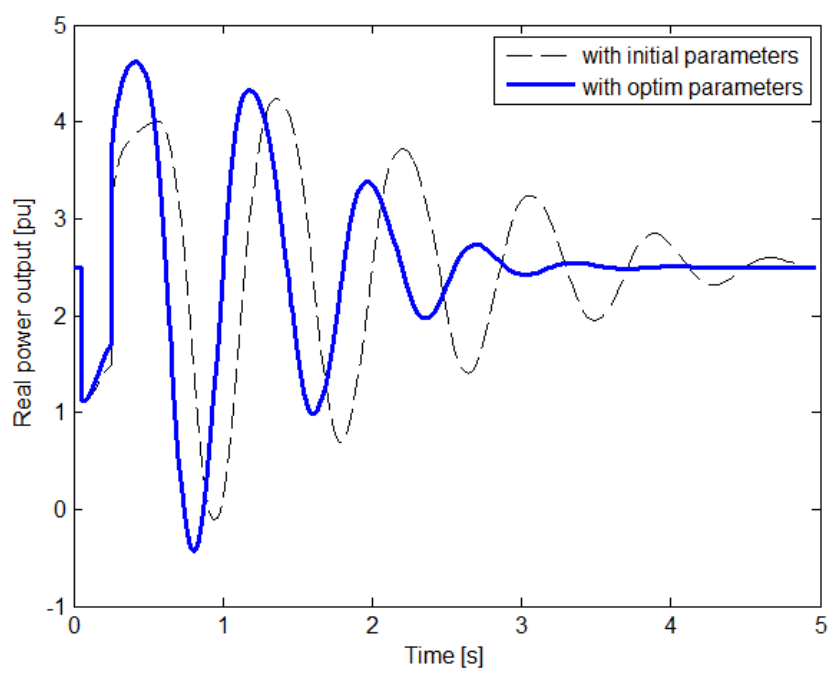

Figure 5. Real Power Response [pu]

\section{Conclusions}

The paper described an optimal parameter selection method of automatic voltage regulator (AVR) for a large power plant in order to improve power system stability. The AVR is generally used to control the output voltage of the generator by regulating the excitation voltage in a generator rotor.

In order to improve transient stability in power system, the power system was modeled exactly in order that the model can have nonlinear behavior. Also, the controllers with AVR and PSS are modeled together with exact power system modeling.

Then, three-phase short circuit was applied to the power system and the responses were investigated. It was clearly shown that the optimal parameters which were obtained with the proposed method in the paper could dramatically improve the transient stability.

\section{Acknowledgements}

This research was supported by Basic Science Research Program through the National Research Foundation of Korea (NRF) funded by the Ministry of Education (NRF2014R1A1A2059165).

\section{References}

[1] G. A. Adepoju and O. A. Lomolafe, "Analysis and Modelling of Static Synchronous Compensator (STATCOM): A comparison of Power Injection and Current Injection Models in Power Flow Study", IJAST, vol.36, (2011).

[2] N. Farouk, "Proposal for Improve the Electrical Power Supply in Port Sudan Town", IJAST, vol.42, (2012).

[3] H. Kouara, H. Laib and A. Chaghi, "A New Method to Extract Reference Currents for Shunt Active Power Filter in Three Phase Four Wire Systems”, IJAST, vol.46, (2012).

[4] B. S Rigby and R. G. Harley, "An improved control scheme for a series-capacitor reactance compensator based on a voltage-source inverter", IEEE Trans. Industry Application, vol.34, (1998), pp.355-363.

[5] P. Kundur, "Power system stability and Control", EPRI Editors, McGraw-Hill, Inc. ISBN 0-07-035958$\mathrm{X}(\mathbf{1 9 9 3 )}$.

[6] S.-M. Baek, J.-W. Park and G.K. Venayagamoorthy, "Power System Control with an Embedded Neural Network in Hybrid System Modeling”, IEEE Trans. on Industry Applications, vol.44, no.5, (2008) pp. 1458-1465. 
[7] S.-M. Baek, J.-W. Park, "RMP Model Based Optimization of Power System Stabilizers in MultiMachine Power System”, Elsevier, Neural Networks, vol.22, no.5-6, (2009), pp.842-850.

[8] S-M. Baek, J-W. Park, and I. A. Hiskens, "Optimal Tuning for Linear and Nonlinear Parameters of Power System Stabilizers in Hybrid System Modeling”, IEEE Trans. on Industry Applications, vol.45, no.1, (2009), pp. 87-97.

[9] A. van der Schaft and H. Schumacher, "An Introduction to Hybrid Dynamical Systems", SpringerVerlag, London, (2000).

[10] S -M. Baek, "Design of PSS and SCRC Controllers to Damp out Low-Frequency Oscillations in Power System", International Journal of Control and Automation, vol.7, no.10, (2014).

[11] B. S. Rigby, N.S. Chonco and R.G. Harley, "Analysis of a power oscillation damping scheme using a voltage-source inverter", IEEE Trans. on Industry Applications, vol.38, no.4, (2002), pp.1105-1113.

[12] J. Nocedal and S. J. Wright, ed., Numerical Optimization, Springer-Verlag, New York, (1999).

\section{Author}

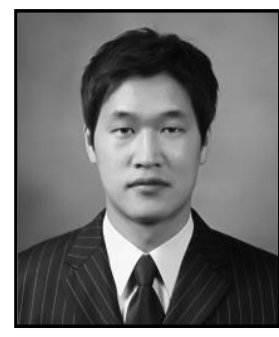

Seung-Mook Baek, he was born in Seoul, Korea. He received B.S., M.S., and Ph.D. degrees from the School of Electrical and Electronic Engineering, Yonsei University, Seoul, Korea, in 2006, 2007, and 2010 respectively, He is currently an Assistant Professor in the Division of Electrical, Electronic and Control Engineering, Kongju National University, Cheonan, Korea. He was a Research Engineer with KEPCO Research Institute, during 2009-2012. His current research interests are in power system dynamics, hybrid systems, optimization control algorithms, realtime simulation, flexible ac transmission system (FACTS) devices, and control of distributed generations. 